\title{
Redes virtuales y apoyo social percibido en usuarios con discapacidad: análisis según la tipología, grado y etapa en la que se adquiere la discapacidad
}

\section{Virtual networks and perceived social support users with disabilities: analysis by type, degree and stage of the disability is acquired}

\author{
Raquel Suriá Martínez \\ Dpto. de Comunicación y Psicología Social \\ Universidad de Alicante, España
}

\begin{abstract}
Resumen
La cantidad de redes sociales online dedicadas a intercambiar apoyo social se ha incrementado en los últimos años. No obstante, el estudio sobre la naturaleza y tipología de estos grupos para jóvenes con discapacidad es escaso. Los objetivos del presente estudio identifican el apoyo social percibido de los jóvenes usuarios de las redes sociales en estos espacios en función del grado, tipología y antigüedad de la discapacidad. Participaron 116 jóvenes con edades comprendidas entre 20 y 35 años (M $=28,22 ;$ DT $=4,02$ ). Fueron clasificados en función de la tipología de la discapacidad (motora, intelectual, visual y auditiva). Contestaron la Escala de apoyo social percibido a través de las redes sociales. Este instrumento está compuesto por una escala de respuestas tipo Likert de cuatro puntos ( $0=$ nada, $4=$ mucho), de 15 ítems repartidos en dos factores. Los resultados muestran mayor percepción de apoyo en las redes sociales online entre los jóvenes con discapacidad motora y con mayor grado de discapacidad. Ello sugiere la importancia que tienen para los jóvenes con discapacidad las redes sociales online para intercambiar apoyo. Así, las redes online podrían servir como ayuda en los programas dirigidos a fomentar la integración social.
\end{abstract}

Palabras clave: jóvenes; discapacidad; redes sociales online; apoyo social percibido.

\begin{abstract}
There has been a recent increase in the number of online social networks for social support exchange. However, studies on the characteristics and types of Internet forums created for young people with disabilities remain scarce. The objective of this study was to identify perceived social support among young users of social networks in these spaces according to the type, degree, and duration of the disability. The study included 116 young people (age range, 20--35 years; $M=28.22, S D=4.02$ ) with different types of disabilities (physical, intellectual, visual, and auditory). They were administered the Perceived Social Support Through Social Networks Scale. This instrument comprises a 4-point Likert scale ( 0 = none, $4=$ a lot) with 15 items divided into two factors. The results show a high level of perceived social support in online social networks among young people with physical disabilities and with greater degrees of disability. These results suggest that online social networks are important to young people with disabilities for social support exchange. They may also be of help in designing programs aimed at promoting social integration.
\end{abstract}

Key words: Young people; Disability; Online social network; Perceived social support.

Cómo citar este artículo: Suriá, R. (2017). Redes virtuales y apoyo social percibido en usuarios con discapacidad: análisis según la tipología, grado y etapa en la que se adquiere la discapacidad. Escritos de Psicología, 10, 31-40.

Correspondencia: Correspondencia: Raquel Suriá Martínez. Dpto. de Comunicación y Psicología Social. Universidad de Alicante, España. E-mail: raquel.suria@ua.es 


\section{Introducción}

La esperanza de vida en las personas con discapacidad ha mejorado de forma importante gracias a los avances médicos y tecnológicos de los últimos años (Gómez, Verdugo y Arias, 2010; Suriá, 2015a).

En los últimos años, el bienestar subjetivo en los jóvenes con discapacidad y sus familias, cada vez cobra mayor importancia (Gomez, et al., 2010). Si se atiende a las cifras del informe realizado por el Instituto Nacional de Estadística (2014), se estima que en España viven 135.100 personas con discapacidad entre 15 y 30 años.

Según la Clasificación Internacional del Funcionamiento, de la Discapacidad y de la Salud (OMS, 2001) estableció que la discapacidad puede ser conceptualizada como toda restricción o ausencia (debida a una deficiencia) de la capacidad de realizar una actividad en la forma o dentro del margen que se considera normal para un ser humano (OMS, 2001), y que, en grado variable, limita algunas de las actividades que pueden realizar el resto de personas, repercutiendo esta situación de forma diferente en la calidad de vida en este colectivo (Riddell y Watson, 2014).

En este sentido, son muchos y variados los determinantes propuestos como factores explicativos del ajuste o la adaptación a la discapacidad. Desde predictores más distales como las variables socioeconómicas, culturales/étnicas y procesos asociados al género hasta determinantes más proximales, como los atributos de personalidad, las valoraciones cognitivas, los estilos de afrontamiento y el apoyo social que percibe la persona (Stanton, Revenson y Tennen, 2007).

Así, la literatura sobre la temática de la discapacidad revela el impacto del apoyo social sobre diferentes procesos relacionados con la discapacidad (Barton, 2015; Pérez y Alcover, 2011; Suriá, 2015a; Verdugo, Schalock, Gómez y Arias, 2007), ya que la presencia de apoyo social se considera como un factor muy importante para prevenir o actuar, por un mecanismo amortiguador, frente el efecto negativo de los acontecimientos vitales estresantes como puede ser vivir con discapacidad (Gifre, del Valle Gómez, Yuguero, Origüen y Bosch, 2010; Palacios, 2008; Vivaldi y Barra, 2012).

Una de las variables más influyentes en la superación exitosa ante el afrontamiento y adaptación de los sucesos complejos que cobran especial importancia en la experiencia de vivir con discapacidad, son las relaciones sociales de las que dispone la persona. Así, el soporte emocional y la ayuda de los demás uno de los soportes fundamentales en el ajuste o adaptación a esta situación (Barton, 2015; Gómez et al., 2010; Morales, Fernández, Infante, Trianes y Cerezo, 2010; Thomas, 2004; Vivaldi y Barra, 2012).

Las fuentes proveedoras de apoyo social más estudiadas son la familia, la red de amigos y los profesionales de salud, influyendo cada una de ellas de diferente forma en la salud del individuo (Dupertuis, Aldwin y Bossé, 2001). Sin embargo, otro tipo de proveedores de relaciones sociales cada vez más frecuentes, que constituye una alternativa generadora de apoyo son las redes sociales online alojadas en internet.

En relación a este aspecto, la literatura existente sobre el tema ha subrayado que los usuarios de las redes virtuales acuden a estos espacios por las numerosas ventajas que les ofrecen. Así, por ejemplo, el rápido intercambio de información, los consejos de autoayuda o el poder compartir las experiencias sobre su problemática con otros usuarios son aspectos que movilizan a participar (Castañeda y Gutiérrez, 2010; Colás-Bravo, González-Ramírez y de Pablos-Pons, 2013; Davison, Pennebaker y Dickerson, 2000; Suriá, 2015b).

Así, existen multitud de grupos online (foros, grupos de discusión, chats, redes sociales, etc.,) que son considerados medios sociales adecuados para favorecer el intercambio de apoyo.

Por ejemplo, Huang y Su (2009), observaron que la participación activa en una comunidad virtual creada para ofrecer apoyo a sus integrantes con discapacidad mental se correlacionaba significativamente con el bienestar subjuetivo de los participantes. En esta misma línea, Välimäki, Hätönen, Lahti, Kuosmanen y Adams, 2012), llevaron a cabo un estudio con pacientes esquizofrénicos. Los resultados indicaron que el grupo que se relacionaba a través de una red social online percibió más apoyo social y menos estrés que el grupo cara a cara.

Al tiempo, estos grupos ofrecen ciertas particularidades como el anonimato, una característica que minimiza la ansiedad que puede suponer una conversación cara a cara (Beléndez y Suriá, 2010; Caplan, 2003; Moya, 2009; Nabi, Prestin y So, 2013; Suriá, 2015b). Esto permite a las personas que padecen enfermedades o condiciones estigmatizadas que perciban la comunicación a través de la red social online, un medio seguro y cómodo, (Suriá y Beléndez, 2011; Sánchez-Franco, Buitrago-Esquinas y Hernández-Mogollón, 2012).

Otros trabajos también han venido a señalar el valor de estos espacios virtuales para traspasar los límites físico-geográficos de las comunidades permitiendo, por un lado, reforzar las relaciones tradicionales, ayudándoles a mantenerlas unidas $\mathrm{y}$, por otro, a desarrollar vínculos con personas con 
intereses y valores comunes que se encuentran lejanas geográficamente (Castelles, 2000; Gabarrón y Fernández-Luque, 2012; Galera, Hurtado y Muñoz, 2014; Malillos, 2011; Suriá, 2015b). Así, por ejemplo, Suriá (2015b) mostró evidencias de la relación positiva encontrada entre el uso de las redes sociales online, las creencias de su uso para incrementar las HHSS y el desarrollo de éstas. Asimismo, la mayoría de jóvenes informó percibir una alta motivación en el uso de estos espacios para sus relaciones personales.

Como se observa en estos estudios, la mayor parte de estas investigaciones han analizado la relación entre el apoyo social percibido y las actitudes y usos de las redes sociales online en usuarios de diferentes entidades diagnósticas (Suriá y Beléndez, 2011) o con enfermedades específicas (Alarcón-Fernández et al., 2011). Asimismo, se ha estudiado el apoyo social en estos grupos en función de la edad (García-Jiménez, López de Ayala-López y Catalina-García, 2013) o del género (Espinar-Ruiz y González-Río, 2009).

Si se echa una mirada a los trabajos referentes a los jóvenes con diferentes tipos de discapacidad y al apoyo social percibido en las redes sociales, si existe literatura científica sobre la percepción de apoyo social en los jóvenes con discapacidad en las redes sociales tradicionales, documentado en éstas, que las características propias de la discapacidad son variables influyentes en el nivel de apoyo percibido en estos grupos (Arribas, 2008; Ballester, Bueno y Sanz-Valero, 2011; García-Ruiz y Quintana, 2015), sin embargo, son inexistentes los trabajos publicados que analicen la relación entre el apoyo percibido en las redes sociales online.

A partir de estas consideraciones, ¿perciben apoyo los jóvenes con discapacidad en las redes sociales online?, ¿qué variables de la discapacidad influyen en la utilización de estos espacios online?, ¿influye la tipología de la discapacidad?, ¿el grado de severidad de la discapacidad de sus usuarios?

Sin duda, profundizar en estas variables de la discapacidad, puede resultar interesante en el estudio de las redes sociales online como otras formas de intercambiar apoyo. Asimismo, otro factor inherente a la discapacidad y que puede tener sentido en este trabajo es la etapa en la que se adquiere la discapacidad. En este sentido, la mayoría de situaciones en las que pensamos que la discapacidad aparece, suponemos que lo más frecuente es que se adquiera en el nacimiento. Sin embargo, hay ocasiones en que el momento vital en el que se detecta o sobreviene la discapacidad es a lo largo de la vida, como por ejemplo sucede al tener una lesión medular como consecuencia de un accidente de tráfico, una enfermedad degenerativa, etc. Sin duda, estas situaciones pueden llegar a desbordar a la persona, no sólo por el shock traumático, sino por el proceso de adaptación y cambio a una nueva forma de vida que debe experimentar tanto el afectado como los que le rodean. Esto podría fomentar la búsqueda de apoyo en mayor medida (Wyndaele y Wyndaele, 2006). De este modo, ¿puede ser la etapa en la que se adquiere la discapacidad una variable influyente en la utilización de las redes sociales online como vía para buscar apoyo?

Con el objetivo de contribuir a resolver estos interrogantes, los objetivos del presente estudio son los siguientes:

1. Conocer el perfil de uso de los jóvenes con discapacidad que utilizan las redes sociales online en busca de apoyo. Esto se examina en función de la tipología, del grado de severidad y de la etapa en la que adquirieron la discapacidad. En este sentido, se espera que:

H1. Existan diferencias significativas en el perfil de uso en función de las variables de los participantes (tipología, del grado de severidad y de la etapa en la que adquirieron la discapacidad).

2. Averiguar el grado de apoyo social que perciben en sus redes sociales online. Esto se examina en función de la tipología, del grado de severidad y de la etapa en la que adquirieron la discapacidad. En concreto se espera que:

H2. Existan diferencias significativas en el apoyo percibido según las variables de los participantes (tipología, del grado de severidad y de la etapa en la que adquirieron la discapacidad).

\section{Participantes}

\section{Método}

Se trata de un estudio exploratorio, éste se llevo a cabo sobre una muestra intencional -por motivos de accesibilidad- de 116 jóvenes con discapacidad, todos ellos procedentes de diversas asociaciones dedicadas a personas con discapacidad, que usuarios de las redes sociales online (ver tabla 1). De ellos, el $53,5 \%$, pertenecen al género femenino y el $46,5 \%$ al masculino, con edades comprendidas entre 20 y 35 años $(M=28,22 ; D T=4,02)$. Fueron clasificados en función de la tipología de la discapacidad que tenían: el 32,5\%, tenía discapacidad motora, el 21,9\% discapacidad intelectual, el 22,8\%, discapacidad visual y el 22,8\% discapacidad auditiva. Asimismo, fueron clasificados en función de 
la etapa en la que adquirieron la discapacidad, siendo el $43,10 \%$ de los jóvenes, con discapacidad adquirida desde el nacimiento y el $56,90 \%$, con discapacidad sobrevenida. Con respecto al grado de discapacidad, el $24,6 \%$ tenía menos del $33 \%$ de discapacidad, un $36,8 \%$ entre el 33 y el $65 \%$ y el $38,6 \%$ más del $65 \%$ de grado de severidad de discapacidad.

Tabla 1

\begin{tabular}{llll} 
Perfil sociodemográfico & & & \\
\hline Perfil sociodemográfico & Mujer & $N$ & $\%$ \\
\hline Sexo & Varón & 61 & 53,5 \\
& $20-23$ & 53 & 46,5 \\
\hline Edad & $24-27$ & 19 & 16,7 \\
& $28-31$ & 40 & 35,1 \\
& $32-35$ & 32 & 28,1 \\
\hline Tipo de discapacidad & Intelectual & 23 & 20,2 \\
& Auditiva & 25 & 21,9 \\
& Motora & 26 & 22,8 \\
& Visual & 37 & 32,5 \\
\hline Etapa de discapacidad & Nacimiento & 26 & 22,8 \\
\hline \multirow{3}{*}{ Grado de severidad de discapacidad } & Menos del $33 \%$ & 50 & 43,10 \\
& Sobrevenida & 66 & 56,90 \\
& Entre 33 y $65 \%$ & 28 & 24,6 \\
& Más del $65 \%$ & 42 & 36,8 \\
\cline { 2 - 4 } & Total & 44 & 38,6 \\
\hline
\end{tabular}

\section{Instrumentos}

Cuestionario sociodemográfico; se diseñó un cuestionario ad hoc para la recogida de los datos sociodemográficos: género, edad, tipología, grado de severidad y etapa en la que adquirieron la discapacidad de los participantes.

Escala de apoyo social percibido a través de las redes sociales, creada por Ramírez, Hernández y Rodríguez, (2013). Esta escala es una adaptación del cuestionario The MOS social support survey (Sherbourne y Stewart, 1991). La escala se compone de 15 ítems. El ítem número 1 hace referencia al tamaño de la red social y los 14 ítems restantes están referidos a cuatro dimensiones del apoyo social funcional: emocional/informacional, instrumental, interacción social positiva y apoyo afectivo. La escala tiene un formato de respuesta tipo Likert (4=mucho, algo, más o menos, casi nada y $0=$ nada). Todos los ítems puntúan de forma positiva. Las pruebas psicométricas del instrumento indican una adecuada consistencia interna de $(a=.94)$ según el alfa de Cronbach. Asimismo, la validez original muestra una varianza explicada del $84.9 \%$, repartida en 2 factores: factor 1: apoyo social percibido (explica el $50 \%$ de varianza) y factor 2: satisfacción con el apoyo social (el cual explica el $34,9 \%$ de varianza).

Con respecto a las propiedades psicométricas del cuestionario en la muestra de este estudio, se calculó la validez a través la técnica del Análisis Factorial Exploratorio de Componentes Principales (Díaz de Rada Igurquiza, 2009), seguido de rotación Varimax para conseguir una mejor comprensión de la matriz resultante, quedando el $60,48 \%$ de la varianza explicada por la escala $(38,20 \%$ de varianza explicada para el primer factor y el $22,28 \%$ de varianza para el segundo factor. En cuanto a la fiabilidad del cuestionario en la muestra de estudio se realizó una evaluación de la consistencia interna mediante el coeficiente Alfa de Cronbach, el cual indicó una consistencia adecuada $(a=, 75)$. Siguiendo el criterio establecido por Nunnally y Bernstein, (1995) se consideran adecuados índices situados entre 0,70 y 0,80 para el cuestionario total.

\section{Procedimiento}

Para recoger los datos se aplicaron las escalas a los participantes. La muestra procedía de diversas asociaciones. Así, para solicitar la colaboración de las personas con discapacidad motora se acudió a la federación de ASPAYM (Asociación de Parapléjicos y Personas con Gran Discapacidad Física). Para los participantes con discapacidad intelectual se acudió a APSA (Asociación pro-discapacitados psíquicos de Alicante). Con objeto de recopilar datos de personas con discapacidad auditiva se acudió a APANAH (Asociación de Padres y Deficientes Auditivos de Alicante). Finalmente, se acudió a la ONCE (Organización Nacional de Ciegos Españoles), para solicitar colaboración a las personas con discapacidad visual. Tras un primer contacto con los directivos de las entidades para explicarles el objetivo del estudio, se propuso la colaboración a los participantes que más jóvenes (entre 18 y 35 años). Para ello, se acudió a algunas de las reuniones que se realizan periódicamente en estas asociaciones y se les 
administró, voluntaria, anónima y presencialmente los cuestionarios. Previamente, otorgaron su consentimiento por escrito. La aplicación del cuestionario se adaptó a las condiciones de cada participante. El tiempo estimado de aplicación fue aproximadamente de 15 minutos para ambos cuestionarios. Los evaluadores fueron previamente entrenados en la aplicación del instrumento. La recopilación de datos se llevó a cabo entre marzo y septiembre del 2015.

\section{Análisis estadístico}

Para los datos sociodemográficos se obtuvieron las frecuencias y porcentajes. Para comprobar si existían diferencias estadísticamente significativas en el apoyo social percibido en las redes sociales online en función de la tipología, el grado y la etapa de la discapacidad se utilizó el análisis de covarianza (ANCOVA). Se controló la covariante edad para eliminar su posible influencia en los resultados.

Finalmente, se realizaron pruebas post hoc para identificar entre qué grupos se encontraban las diferencias. Se utilizó el método de Scheffé al no estar cada grupo compuesto por el mismo número de participantes. Los datos se analizaron por medio del paquete estadístico SPSS versión 19.0.

\section{Resultados}

Al atender al primer objetivo, conocer el perfil de uso de los jóvenes con discapacidad que utilizan las redes online en busca de apoyo en función de la tipología, del grado de severidad y de la etapa en la que adquirieron la discapacidad (tablas 2 y 3 ), se observa que, en general, la mayoría de los participantes entra en las redes sociales online varias veces al día $(54,3 \%)$, durante al menos 1 hora y 2 horas al día $(34,5 \%)$.

Así, al examinar las posibles diferencias en el perfil de utilización de las redes sociales online en función de las variables relacionadas con la discapacidad analizadas en el estudio, se observan los siguientes resultados:

En referencia a la frecuencia de visitas, los participantes con discapacidad motora indican entrar con mayor frecuencia que los otros grupos $\left[\chi^{2}{ }_{(9)}=15,772, p<, 05\right]$. No se observan diferencias estadísticamente significativas ni en función del grado de severidad de la discapacidad, ni de la etapa en la que adquirieron ésta (tabla 2).

Tabla 2

Perfil de frecuencia de utilización de las redes sociales online

\begin{tabular}{|c|c|c|c|c|c|c|c|c|c|c|c|c|}
\hline & & \multicolumn{10}{|c|}{ ¿Con qué frecuencia entras en las redes sociales online? } & \multirow{3}{*}{$\begin{array}{c}\text { Sig. } \\
\text { estadística } \\
\chi^{2}\end{array}$} \\
\hline & & \multicolumn{2}{|c|}{$\begin{array}{c}1 \text { alguna vez al } \\
\text { mes }\end{array}$} & \multicolumn{2}{|c|}{ Una vez al día } & \multicolumn{2}{|c|}{$\begin{array}{c}\text { Varias veces } \\
\text { semana }\end{array}$} & \multicolumn{2}{|c|}{ Varias veces al día } & \multirow[b]{2}{*}{$N$} & \multirow[b]{2}{*}{$\%$} & \\
\hline & & $N$ & $\%$ & $N$ & $\%$ & $N$ & $\%$ & $N$ & $\%$ & & & \\
\hline \multirow{4}{*}{ Tipo disc. } & Intelectual & 3 & 11,10 & 13 & 48,10 & 4 & 14,80 & 7 & 25,90 & 27 & 100,00 & \multirow{4}{*}{$15,77^{*}$} \\
\hline & Auditiva & 0 & 0,00 & 5 & 23,80 & 10 & 46,00 & 6 & 30,20 & 21 & 100,00 & \\
\hline & Motora & 3 & 6,30 & 11 & 22,90 & 6 & 12,50 & 28 & 58,30 & 48 & 100,00 & \\
\hline & Visual & 1 & 5,00 & 5 & 25,00 & 2 & 10,00 & 12 & 60,00 & 20 & 100,00 & \\
\hline \multirow{3}{*}{ Grado disc. } & Menos del 33\% & 3 & 6,80 & 10 & 22,70 & 8 & 18,20 & 23 & 52,30 & 44 & 100,00 & \multirow{3}{*}{12,43} \\
\hline & Del $33 \%$ al $65 \%$ & 2 & 7,10 & 10 & 35,70 & 2 & 7,10 & 14 & 50,00 & 28 & 100,00 & \\
\hline & Más del $65 \%$ & 2 & 4,50 & 14 & 31,80 & 2 & 4,50 & 26 & 59,10 & 44 & 100,00 & \\
\hline \multirow{3}{*}{ Etapa disc. } & Nacimiento & 3 & 6,00 & 19 & 38,00 & 4 & 8,00 & 24 & 48,00 & 50 & 100,00 & \multirow[t]{2}{*}{,224 } \\
\hline & Sobrevenida & 4 & 6,10 & 15 & 22,70 & 8 & 12,10 & 39 & 59,10 & 66 & 100,00 & \\
\hline & Total & 7 & 6,00 & 34 & 29,30 & 12 & 10,30 & 63 & 54,30 & 116 & 100,00 & \\
\hline
\end{tabular}

$\left({ }^{*}\right)=$ Nivel de Significación de $.001 ;\left(^{*}\right)=$ Nivel de Significación de .05 .

Con respecto al tiempo que pasan en la red social online (tabla 3), los resultados siguen la misma dirección que los referentes a la frecuencia de acceso, son los participantes con discapacidad motora los que indican entrar con mayor frecuencia que los otros grupos $\left[\chi^{2}{ }_{(9)}=12,244, p<, 05\right]$. No se observan diferencias estadísticamente significativas ni en función del grado de severidad de la discapacidad, ni de la etapa en la que adquirieron ésta. 
Tabla 3

Tiempo conectado a la red social online

\begin{tabular}{|c|c|c|c|c|c|c|c|c|c|c|c|c|}
\hline \multicolumn{13}{|c|}{ ¿Cuánto tiempo pasas en la red social online? } \\
\hline & & \multicolumn{2}{|c|}{$\begin{array}{l}\text { Menos de } 15 \\
\text { minutos }\end{array}$} & \multicolumn{2}{|c|}{$\begin{array}{c}\text { Entre } 15 \text { y } 1 \\
\text { hora }\end{array}$} & \multicolumn{2}{|c|}{$\begin{array}{c}\text { Entre } 1 \text { hora y } 2 \\
\text { horas }\end{array}$} & \multicolumn{2}{|c|}{ Más de 2 horas } & \multicolumn{2}{|r|}{ Total } & \multirow{2}{*}{$\frac{\text { Sig. estadística }}{\chi^{2}}$} \\
\hline & & $N$ & $\%$ & $N$ & $\%$ & $N$ & $\%$ & $N$ & $\%$ & $N$ & $\%$ & \\
\hline \multirow{4}{*}{ Tipo disc. } & Intelectual & 7 & 25,90 & 10 & 37,00 & 10 & 37,00 & 0 & 0,00 & 27 & 100,00 & \multirow{4}{*}{$12,244^{*}$} \\
\hline & Auditiva & 2 & 9,50 & 7 & 33,30 & 6 & 28,60 & 6 & 28,60 & 21 & 100,00 & \\
\hline & Motora & 6 & 12,50 & 9 & 18,80 & 17 & 35,40 & 16 & 33,30 & 48 & 100,00 & \\
\hline & Visual & 6 & 30,00 & 3 & 15,00 & 7 & 35,00 & 4 & 20,00 & 20 & 100,00 & \\
\hline \multirow{3}{*}{ Grado disc. } & Menos del 33\% & 8 & 18,20 & 11 & 25,00 & 15 & 34,10 & 10 & 22,70 & 44 & 100,00 & \multirow{3}{*}{2,239} \\
\hline & Del $33 \%$ al $65 \%$ & 6 & 21,40 & 8 & 28,60 & 8 & 28,60 & 6 & 21,40 & 28 & 100,00 & \\
\hline & Más del $65 \%$ & 5 & 11,40 & 10 & 22,70 & 17 & 38,60 & 12 & 27,30 & 44 & 100,00 & \\
\hline \multirow{3}{*}{ Etapa disc. } & Nacimiento & 10 & 20,00 & 13 & 26,00 & 13 & 26,00 & 14 & 28,00 & 50 & 100,00 & \multirow{2}{*}{3,115} \\
\hline & Sobrevenida & 9 & 13,60 & 16 & 24,20 & 27 & 40,90 & 14 & 21,20 & 66 & 100,00 & \\
\hline & Total & 19 & 16,4 & 29 & 25,0 & 40 & 34,5 & 28 & 24,1 & 116 & 100,0 & , \\
\hline
\end{tabular}

$\left({ }^{* *}\right)=$ Nivel de Significación de $.001 ;\left(^{*}\right)=$ Nivel de Significación de .05 .

En relación al segundo objetivo, averiguar el grado de apoyo social que perciben en sus redes sociales online en función de la tipología, del grado de severidad y de la etapa en la que adquirieron la discapacidad, se observan los siguientes resultados:

Al examinar las puntuaciones medias de los participantes en la escala global de apoyo (tabla 4), se observa que presentan un nivel de apoyo percibido notable ( $0=$ nada, $4=$ mucho).

Al atender a las puntuaciones de la escala según la tipología de la discapacidad, los resultados indican diferencias estadísticamente significativas tanto en la escala global $\left[F_{(3,112)}=3,30, p=, 023\right]$, como en ambos factores de la escala, observándose que el grupo de participantes con discapacidad motora es el que indica puntuaciones medias más elevadas que los jóvenes con los otros tipos de discapacidad (ver tabla 4). No se observa influencia de la covariante edad $(I=0,097, F=2,100, p=, 978)$.

En relación al apoyo social percibido en función del grado de severidad de la discapacidad el análisis de varianza indica diferencias estadísticamente significativas entres las puntuaciones medias de los grupos tanto en la escala general $\left[F_{(2,112)}=2,435, p=, 047\right]$, como en ambos factores (tabla 4), observándose que el grupo con mayor grado de severidad de discapacidad indica percibir más apoyo en esto espacios online. No se observa influencia de la covariante edad $(I=0,078, F=2,100, p=, 682)$.

No se observan diferencias con respecto al apoyo percibido en función de la etapa en la que adquirieron la discapacidad, ni en la escala total ni en los factores componentes de la escala de apoyo. Del mismo modo, la edad no parece afectar al apoyo percibido $(I=0,118, F=2,100, p=, 486)$.

Tabla 4

Puntuaciones medias en la escala de apoyo social percibido según tipología, grado y antigüedad de discapacidad

\begin{tabular}{|c|c|c|c|c|c|c|c|c|}
\hline & & \multicolumn{3}{|c|}{$\begin{array}{c}\text { F1 } \\
\text { Apoyo social percibido }\end{array}$} & \multicolumn{2}{|c|}{$\begin{array}{c}\text { F2 } \\
\text { satisfacción con el apoyo social }\end{array}$} & \multicolumn{2}{|c|}{ Total } \\
\hline & & $N$ & $M$ & $D T$ & $M$ & $D T$ & $M$ & $D T$ \\
\hline \multirow{6}{*}{ Tipo disc. } & Intelectual & 27 & 16,30 & 4,25 & 19,70 & 6,50 & 36,00 & 10,46 \\
\hline & Auditiva & 21 & 17,81 & 3,19 & 20,52 & 3,87 & 38,33 & 6,53 \\
\hline & Motora & 48 & 19,60 & 3,65 & 22,88 & 4,85 & 42,48 & 8,31 \\
\hline & Visual & 20 & 16,60 & 5,53 & 20,55 & 7,56 & 37,15 & 12,86 \\
\hline & Total & 116 & 17,99 & 4,29 & 21,31 & 5,75 & 39,30 & 9,77 \\
\hline & Sig. Estadís. & & $F=4,805$ & $p=, 003$ & $F=2,200$ & $p=, 092$ & $F=3,300$ & $p=8,36$ \\
\hline \multirow{5}{*}{ Grado disc. } & Menos del 33\% & 44 & 16,84 & 3,70 & 19,93 & 5,12 & 36,77 & 9,82 \\
\hline & Del $33 \%$ al $65 \%$ & 28 & 18,68 & 4,49 & 22,18 & 5,54 & 40,86 & 10,69 \\
\hline & Más del $65 \%$ & 44 & 18,70 & 5,06 & 22,14 & 6,32 & 40,84 & 9,77 \\
\hline & Total & 116 & 17,99 & 4,29 & 21,31 & 5,75 & 39,30 & 9,03 \\
\hline & Sig. Estadís. & & $F=2,615$ & $p=, 078$ & $F=2,074$ & $p=, 130$ & $F=2,435$ & $p=0,47$ \\
\hline \multirow{4}{*}{ Etapa disc. } & Nacimiento & 50 & 17,44 & 4,28 & 21,20 & 5,07 & 38,64 & 10.33 \\
\hline & Sobrevenida & 66 & 18,41 & 4,29 & 21,39 & 6,26 & 39,80 & 10,41 \\
\hline & Total & 116 & 17,99 & 4,29 & 21,31 & 5,75 & 39,30 & 9,03 \\
\hline & Sig. Estadís. & & $F=-1,206$ & $p=, 114$ & $F=, 230$ & $p=, 102$ & $F=1,002$ & $p=, 108$ \\
\hline
\end{tabular}

$\left({ }^{* *}\right)=$ Nivel de Significación de $.001 ;\left(^{*}\right)=$ Nivel de Significación de .05 . 


\section{Discusión}

En este trabajo se pretende reflejar la importancia que tiene para los jóvenes con discapacidad las redes sociales online a la hora de percibir apoyo. Así, tal y como se comprueba en nuestros resultados, podemos observar que este recurso es utilizado por la mayoría de jóvenes, y no tan jóvenes, que viven una discapacidad, frecuentando gran parte de ellos estos espacios con asiduidad y dedicándole bastante tiempo al día.

Esto refleja que, aunque es evidente que estos espacios son utilizados por usuarios sin ningún tipo de discapacidad, para algunos jóvenes con determinadas características, como vivir con discapacidad, se hace un recurso especialmente útil que, va a aportarles la posibilidad de reducir sus limitaciones e incrementar las vías de interacción y apoyo.

Esto se reafirma en mayor medida en los jóvenes con discapacidades específicas, como sucede con los jóvenes con discapacidad motora. Estos resultados confirmarían la primera hipótesis del estudio relativa a la existencia de diferencias significativas en el perfil de uso en función de las variables de los participantes, en este caso, la tipología de la discapacidad.

Esto por tanto, evidencia que la movilidad reducida limita en gran medida, las posibilidades de desplazamiento de los afectados, con ello, de disponer de menos oportunidades para interaccionar con sus iguales y por tanto, los beneficios que puede aportarles disponer de otras formas de interacción accesibles, como las redes sociales online.

En esta línea, estos resultados son respaldados por otros estudios (Eden y Seiman, 2011; Suriá, 2012; 2015b; Zubillaga-del Río y Alba-Pastor, 2013) que indican preferencia hacia las redes sociales online en muchos jóvenes con características concretas, como vivir con movilidad reducida.

A su vez, estos datos guardan relación con los obtenidos al atender a los resultados del siguiente objetivo, conocer el apoyo social que perciben los jóvenes con discapacidad. Así, de forma general, las puntuaciones medias de la escala de apoyo social muestran que los participantes indican un alto nivel de apoyo social percibido en estos espacios. Tal y como diferentes autores han enfatizado, estos espacios online son muy atractivos para ciertos colectivos, y más concretamente para jóvenes con discapacidad ya que, características como la eliminación de barreras, la facilidad para acceder a cualquier hora pueden ayudar a fomentar la interacción con otros compañeros de estudios y/o trabajo, amigos y usuarios de las redes sociales online (Caplan, 2003; Livingstone, 2008; Suriá y Beléndez, 2011; Suriá, 2015b; Zubillaga-del Río y Alba-Pastor, 2013) al mitigar o reducir las barreras derivadas de su discapacidad.

Con respecto al apoyo social percibido por los usuarios en las redes sociales online en función del grado de severidad de la discapacidad los resultados indican que a mayor grado de discapacidad, mayor percepción de apoyo social. Esto sugiere que las limitaciones de la movilidad reducida serían una de las principales razones por las que los jóvenes con discapacidad entran en estos espacios en busca de apoyo.

Estos resultados quedan respaldados al atender a la siguiente cuestión referente al nivel de apoyo social percibido en función de la tipología de la discapacidad de los participantes, reflejándose en los resultados que los usuarios con discapacidad motora muestran mayores puntuaciones en a escala, es decir, son los que más apoyo social perciben en estos espacios virtuales. Estos resultados confirmarían la segunda hipótesis del estudio, la existencia de diferencias estadísticamente significativas en el apoyo percibido en las redes sociales online en función de las características de los participantes, en este caso, en función de la tipología y del grado de severidad de la discapacidad.

Como en líneas anteriores se ha mencionado, las personas con discapacidad motora ven más reducidas sus posibilidades de desplazamiento, esto podría limitar la cantidad y la calidad de las experiencias sociales vividas por las personas con discapacidad y con ello, disponer de menos oportunidades para obtener apoyo de las relaciones tradicionales o "cara a cara".

Esto va en línea con lo indicado por otros autores (Caplan, 2003; Suriá et al., 2015; Valkenburg, Peter y Schouten, 2006), que han señalado que en ocasiones, las personas afectadas por alguna discapacidad motora pueden carecer de estrategias, habilidades y competencias para establecer relaciones sociales satisfactorias como consecuencia de las actitudes de sobreprotección de los adultos, la carencia de relaciones sociales y la falta de conocimiento social, relacionados con las limitaciones funcionales derivadas de la propia discapacidad. Esto desemboca en un mayor riesgo en la restricción de su participación en algunas actividades, especialmente las que exigen esfuerzo físico, y en un mayor temor al fracaso o rechazo social.

A la luz de los resultados obtenidos, se deduce que las redes sociales online pueden ser una vía fructífera de apoyo para los jóvenes con discapacidad, especialmente, jóvenes con discapacidad motora. Sin embargo, el trabajo presenta algunas limitaciones que es necesario tener en cuenta. La 
principal radica en la tipología de la discapacidad, evidentemente, ésta es única para cada persona ya que está influida por una compleja combinación de factores (desde las diferencias personales de experiencias, temperamentos y contextos diferentes), por tanto, deberíamos profundizar en multitud de variables que quedan por tener en cuenta y que podían explicar la variabilidad en la utilización y en el apoyo percibido tanto dentro de las redes online como en las relaciones cara a cara.

Asimismo, aspectos de la metodología como el reducido número de participantes limitan los resultados. Pese a la complejidad de salvar esta limitación, por la dificultad de acceder a una muestra más elevada, en futuras investigaciones se debería controlar este factor ampliando el estudio a una muestra más elevada y representativa de jóvenes con discapacidad con el objeto de incrementar la validez interna de los resultados.

Del mismo modo, sería interesante añadir una medida de bienestar psicológico a de adaptación personal y/o social para conocer las ventajas en el bienestar emocional y social del apoyo social percibido. Finalmente, podría ser fructífera la comparación de la utilización de las redes por los jóvenes que no viven la experiencia de tener una discapacidad y su percepción de estos espacios como vía para el intercambio de apoyo.

\section{Referencias}

1. Alarcón-Fernández, O., Alonso-Abreu, I., Carrillo-Palau, M., Nicolás-Pérez, D., Gimeno, A. Z., Ramos, L., ... y Quintero-Carrión, E. (2011). Uso de Internet entre los pacientes con enfermedades digestivas en un hospital general. Gastroenterología y Hepatología, 34(10), 667-671. https:// doi.org/10.1016/j.gastrohep.2011.08.006

2. Arribas, F. J. R. (2008). Espacios sociales de participación: las asociaciones y los grupos de autoayuda. Nómadas: revista crítica de ciencias sociales y jurídicas, (18), 307-314.

3. Ballester, R., Bueno, E. y Sanz-Valero, J. (2011). Información, autoayuda y creación de identidades: las tecnologías de la información y la comunicación (TIC) y las asociaciones de discapacitados físicos. El ejemplo de la poliomielitis. Salud colectiva, 7, 539-547.https://doi. org/10.18294/sc.2011.389

4. Barton, L. (2015). Estudios sobre discapacidad y la búsqueda de inclusividad: observaciones. Revista de Educación, 349, 137-152

5. Beléndez, M. y Suriá, R. (2010). Apoyo a un «click» de ratón: los foros de Internet para problemas de salud. Acción psicológica, 7(1), 17-29. https://doi.org/10.5944/ap.7.1.202

6. Caplan, S. E. (2003). Preference for online social interaction a theory of problematic Internet use and psychosocial well-being. Communication research, 30(6), 625-648. https://doi. org/10.1177/0093650203257842

7. Castelles, M. (2000). The Information Society: The Power of Identity. Blackwell: Oxford.

8. Castañeda, L. y Gutiérrez, I. (2010). Redes sociales y otros tejidos online para conectar personas. Aprendizaje con redes sociales. Tejidos educativos para los nuevos entornos, 17-39.

9. Colás-Bravo, P., González-Ramírez, T. y de Pablos-Pons, J. (2013). Juventud y redes sociales: Motivaciones y usos preferentes. Comunicar: Revista Científica de Comunicación y Educación, 20(40), 15-23. https://doi.org/10.3916/C40-2013-02-01

10. Davison, K.P., Pennebaker, J.W. y Dickerson, S.S. (2000). Who talks? The social psychology of illness groups. American Psychologist, 55(2), 205-217. https://doi.org/10.1037/0003-066X.55.2.205

11. de Rada Igúzquiza, V. D. (2009). Análisis de datos de encuestas: Desarrollo de una investigación completa utilizando SPSS (Vol. 137). Barcelona: Editorial UOC.

12. DuPertuis, L., Aldwin, C. y Bosse, R. (2001). Does the source of support matter for different health outcomes? Findings from the Normative Aging Study. Journal of Aging and Health, 13, 494-510. https://doi.org/10.1177/089826430101300403

13. Eden, S. y Heiman, T. (2011). Computer mediated communication: Social support for students with and without learning disabilities. Educational Technology \& Society, 14, 89-97.

14. Espinar Ruiz, E. y González Río, M. J. (2009). Jóvenes en las redes sociales virtuales: un análisis exploratorio de las diferencias de género. Feminismo/s, (14), 87-106. https://doi.org/10.14198/fem.2009.14.06

15. Gabarrón, E. y Fernández-Luque, L. (2012). eSalud y vídeos online para la promoción de la salud. Gaceta Sanitaria, 26(3), 197-200. https://doi.org/10.1016/i.gaceta.2012.03.004

16. Galera, M. D. C. G., Hurtado, M. D. H. y Muñoz, C. F. (2014). Las redes sociales en la cultura digital: percepción, participación, movilización. Revista de la Asociación Española de Investigación de la Comunicación, 1, 1-6. 
17. García-Jiménez, A., López de Ayala-López, M. C. y Catalina-García, B. (2013). Hábitos de uso en Internet y en las redes sociales de los adolescentes españoles. Comunicar: Revista Científica de Comunicación y Educación, 21(41), 195-204. https://doi.org/10.3916/C41-2013-19

18. García-Ruiz, S. y Quintana, P. (2015). Las redes de los afectos: aprendizajes en la Red de Rehabilitación Basada en Comunidad de las Américas. Revista de Facultad de Medicina, 63, 161-168. https://doi.org/10.15446/revfacmed.v63n3sup.50569

19. Gifre, M., del Valle Gómez, A., Yuguero, M., i Origüen, Á. G. y Bosch, P. M. (2010). La mejora de la calidad de vida de las personas con lesión medular: La transición del centro rehabilitador a la vida cotidiana desde la perspectiva de los usuarios. Athenea Digital: revista de pensamiento e investigación social, (18), 3-15.

20. Gómez, L. E., Verdugo, M. Á. y Arias, B. (2010). Calidad de vida individual: avances en su conceptualización y retos emergentes en el ámbito de la discapacidad. Psicología Conductual, 18(3), 453.

21. Huang, Y-h. y Su, I-J. (2009). Does Health Related Quality of Life Differ between People with Chronic Mental IIIness Who Use Computers and Those Who Do Not? Universal Access in HCl,1, 361-366. https://doi.org/10.1007/978-3-642-02707-9_41

22. Instituto Nacional de Estadística, INE. (2014). Panorámica de la discapacidad en España. Recuperado de: http://www.ine.es/buscar/searchResults.do?searchStrin=discapacidad\&Menu b

23. Livingstone, S. (2008). Taking risky opportunities in youthful content creation: teenagers' use of social networking sites for intimacy, privacy and self-expression. New media and society, 10, 393411. https://doi.org/10.1177/1461444808089415

24. Malillos, L. M. (2011). Jóvenes en redes sociales: significados y practicas de una sociabilidad digital. Revista de Estudios de Juventud, 95, 31-43.

25. Morales, F. M., Fernández, F. J., Infante, L., Trianes, M. V. y Cerezo, M. T. (2010). Eficacia de una intervención para incrementar apoyo social en adolescentes. Revista Latinoamericana de Psicología, 41(1), 141-152.

26. Moya, A. M. (2009). Las nuevas tecnologías en la educación. Revista Digital Innovación y experiencias educativas, (24). Recuperado el 20 de mayo de 2015, de:http://www.csisif.es/andalucia/ modules/mod ense/revista/pdf/Numero 24/ANTONIA M MOYA 1.pdf

27. Nabi, R. L., Prestin, A. y So, J. (2013). Facebook friends with (health) benefits? Exploring social network site use and perceptions of social support, stress, and well-being. Cyberpsychology, Behavior, and Social Networking, 16(10), 721-727. https://doi.org/10.1089/cyber.2012.0521

28. Nunnally, J.C. y Bernstein, I.J. (1995). Teoría psicométrica. Madrid: McGraw-Hill.

29. OMS (2001). Clasificación Internacional del Funcionamiento de la Discapacidad y de la Salud. Madrid: Ministerio de Trabajo y Asuntos Sociales.

30. Palacios, A. (2008). El modelo social de discapacidad: orígenes, caracterización y plasmación en la Convención Internacional sobre los Derechos de las Personas con Discapacidad. Madrid: Cermi.

31. Pérez, V. y Alcover, C. (2011). Apoyo social, satisfacción laboral y abandono en trabajadores con discapacidad. Boletín de Psicología, 102, 23-41.

32. Ramírez, M. T. G., Hernández, R. L. y Rodríguez, C. L. D. (2013). Propiedades psicométricas de una escala para evaluar el apoyo social a través de las redes sociales. Acta de investigación psicológica, 3(3), 1238-1247. https://doi.org/10.1016/S2007-4719(13)70963-3

33. Riddell, S. y Watson, N. (2014). Disability, culture and identity. Routledge.

34. Sánchez-Franco, M. J., Buitrago-Esquinas, E. M. y Hernández-Mogollón, J. M. (2012). Antecedentes sociales y psicológicos del compromiso comunitario. Un análisis del comportamiento del usuario de una red social de relaciones. Cuadernos de Economía y Dirección de la Empresa, 15(4), 205-220. https://doi.org/10.1016/j.cede.2012.04.007

35. Sherbourne, C.D. y Stewart, A.L. (1991).The MOS Social Support Survey. Social Science and Medicine, 32(6), 705-714. https://doi.org/10.1016/0277-9536(91)90150-B

36. Stanton, A. L., Revenson, T. A. y Tennen, H. (2007). Health psychology: psychological adjustment to chronic disease. Annu. Rev. Psychology, 58, 565-592. https://doi.org/10.1146/annurev. psych.58.110405.085615

37. Suriá, R. (2015a). Perfiles resilientes y calidad de vida en personas con discapacidad sobrevenida por accidentes de tráfico. Gaceta Sanitaria, 29, 55-59. https://doi.org/10.1016/j. gaceta.2015.01.016

38. Suriá, R. (2015b). Jóvenes con discapacidad motora y redes sociales online, $¿$ nuevos espacios para el desarrollo de habilidades sociales. Anuario de psicología, 45(1), 71-85. 
39. Suriá, R., Gracía-Fernández, J. M. y Ortigosa, J. M. (2015). Perfiles resilientes y su relación con las habilidades sociales en personas con discapacidad motora. Psicología Conductual, 23(1), 35.

40. Suriá, R. y Belendez, M. (2011). Grupos de apoyo virtuales dedicados a problemas de salud: estudio de su tipología y análisis de su representatividad. Anales de psicología, 27(1), 210-220.

41. Thomas, C. (2004). Disability and impairment. J. Swain et al., Hrsg, 2, 21-27.

42. Välimäki, M., Hätönen, H., Lahti, M., Kuosmanen, L. y Adams, C. E. (2012). Information and communication technology in patient education and support for people with schizophrenia. Cochrane Database of Systematic Reviews, 10. Recuperado de: http://www.update-software.com/BCP/ WileyPDF/EN/CD007198.pdf. https://doi.org/10.1002/14651858.CD007198.pub2

43. Valkenburg, P. M., Peter, J. y Schouten, A. P. (2006). Friend networking sites and their relationship to adolescents' well-being and social self-esteem. CyberPsychology \& Behavior, 9(5), 584-590. https://doi.org/10.1089/cpb.2006.9.584

44. Verdugo, M. A., Schalock, R. L., Gómez, I. E. y Arias, B. (2007). Construcción de escalas de calidad de vida multidimensionales centradas en el contexto: la Escala GENCAT. Siglo Cero, 38(224), 57-72.

45. Vivaldi, F. y Barra, E. (2012). Bienestar psicológico, apoyo social percibido y percepción de salud en adultos mayores. Terapia psicológica, 30(2), 23-29. https://doi.org/10.4067/S071848082012000200002

46. Wyndaele, M. y Wyndaele, J. (2006). Incidence, prevalence and epidemiology of spinal cord injury: What learns a worldwide literature survey? Spinal Cord, 44(9), 523-529. https://doi.org/10.1038/ sj.sc. 3101893

47. Zubillaga-del Río, A. y Alba-Pastor, C. (2013). La discapacidad en la percepción de la tecnología entre estudiantes universitarios. Comunicar: Revista Científica de Comunicación y Educación, 20(40), 165-172.

RECIBIDO: 03 MARZO 2016

MODIFICADO: 26 ENERO 2017

ACEPTADO: 14 marzo 2017 